Communication

\title{
Cognitive Reserve: Cognitive Abilities that Shield Against Dementia Symptomatology
}

\author{
Francisca S. Rodriguez (Then) ${ }^{1,2,3, *}$
}

1. Center for Cognitive Science, University of Kaiserslautern, Kaiserslautern, Germany

2. Edward R. Roybal Institute on Aging, University of Southern California, United States

3. Institute of Social Medicine, Occupational Health and Public Health, University of Leipzig, Germany

* Correspondence: Francisca S. Rodriguez; E-Mail: frodrigu@rhrk.uni-kl.de

Academic Editor: Michael Fossel

\section{OBM Geriatrics}

2018, volume 2 , issue 4

doi:10.21926/obm.geriatr.1804017
Received: June 19, 2018

Accepted: October 19, 2018

Published: October 31, 2018

\begin{abstract}
Background: Cognitive reserve describes the capacity for cognitively normal functioning despite brain damage. Previous research on cognitive reserve outlined how characteristics of the brain promote good cognitive functioning, although it is not yet clear what cognitive processes help individuals to maintain good cognitive functioning in the face of brain damage.
\end{abstract}

Methods: Narrative review of relevant articles identified in literature searches for cognitive and/or psychometric characteristics of cognitive reserve.

Results: The existing evidence on cognitive processes of cognitive reserve is extremely sparse. Results from a diverse set of studies suggest that cognitive reserve may comprise verbal memory and executive functions.

Conclusions: We propose that cognitive reserve consists of a combination of elaborate networks of mental representations and proficient executive functioning. Future studies are required to validate the proposed mechanism of cognitive reserve.

\section{Keywords}

Cognitive reserve; Alzheimer's disease; dementia; memory; executive functioning

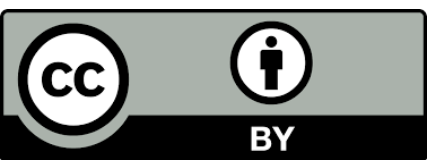

(C) 2018 by the author. This is an open access article distributed under the conditions of the Creative Commons by Attribution License, which permits unrestricted use, distribution, and reproduction in any medium or format, provided the original work is correctly cited. 


\section{Introduction}

Cognitive reserve refers to a puzzling discrepancy between observed and expected cognitive function considering underlying brain pathology $[1,2]$. Based on the information obtained using currently available methods, the capacity to maintain good cognitive function cannot be explained by the level of brain pathology. For instance, brain scans show significant brain atrophy although cognitive functioning scores fall within the normal range. To date, the cognitive processes that help some individuals to maintain a better level of functioning have not been identified. Barulli and Stern (2013) emphasized that "identifying the particular cognitive strategies or compensatory mechanisms [of cognitive reserve] may help to identify the underlying neural mechanisms, as well as point towards effective interventions" [3]. Previous research focused mainly on investigation of the neurobiological differences between individuals with lower and higher cognitive functioning [4, 5] to define the characteristics of brain reserve and neural reserve. Here, we discuss the scientific literature on cognitive differences with the aim of providing an improved understanding of the cognitive processes involved in cognitive reserve. Since, by definition, cognitive reserve is 'software' that operates on a neural basis [1], it is essential to understand the cognitive processes involved. The available evidence on cognitive processes indicates that cognitive reserve may be the result of a combination of elaborate networks of mental representations and proficient executive functioning.

\section{Literature Search Process}

A literature search of the PubMed, PsycINFO, and Google Scholar databases was performed in February 2017. Search terms included 'cognitive reserve', 'brain reserve', and 'neural reserve'. No further terms or filters were applied. Relevant studies were categorized as either 'reviews', including any type of review or meta-analysis or 'primary evidence studies', including experiments and longitudinal and cross-sectional studies. In addition, manual searches were performed based on references from identified studies and to identify further studies by authors working on cognitive reserve. After an initial title/abstract screening, a total of 78 full-text ( $n=24$ reviews; $n=$ 41 primary evidence studies; $n=13$ manual search) were identified as relevant regarding the provision of information on cognitive reserve. There is no standardized way to assess cognitive reserve. Cognitive reserve was usually assessed on the basis of level of education, sometimes in combination with other markers such as occupation and reading level. However, every study provided valuable information on trajectories of cognitive functioning with respect to cognitive reserve.

\section{Brain, Neural, and Cognitive Reserve}

The brain pathology found in patients with dementia normally leads to the corresponding clinical symptomatology; however, some individuals seem better able to compensate for brain pathology. Brain, neural, and cognitive reserves are thought to contribute to resilience against developing clinical symptoms in the face of brain pathology. Brain reserve describes differences in 
quantitative aspects of the brain [3], such as weight, number of neurons or synapses [6], and intracranial volume [7]. Several studies have shown that a higher level of education is associated with these quantitative aspects of the brain. A review by Morbelli and Nobili (2014) summarized the findings of previous studies showing that a higher level of education is associated with increased regional cortical thickness in healthy controls but also inversely correlated with regional cortical thickness in patients with Alzheimer's disease (AD) [8]. A review by Bartres-Faz and Arenaza-Urquijo (2011) further discussed the association between education and a reduced intracranial volume, smaller head size, and the rate of hippocampal atrophy [5]. Neural reserve (quantitative aspects of the brain at the neural level) additionally promotes efficient brain functioning [9]. For instance, individuals with a higher level of education seem to have diminished age-related alterations in cerebrospinal fluid (CSF) biomarkers of AD [9], such as a lower amyloid load [10], which is important for preservation of synaptic plasticity [11]. However, the state of the brain does not explain why some individuals, such as those with a higher level of education, do not show clinical symptoms of dementia. The capacity to 'withhold' clinical symptomatology and to maintain a good cognitive functioning is known as 'cognitive reserve' [1].

To understand cognitive reserve, researchers explored functional processes in the brain. These functional processes are also referred to as neural compensation because it appears that individuals with high cognitive reserve recruit alternative networks to compensate for the pathological disruption $[6,12]$. Research findings demonstrate that cognitive reserve draws on the ventral and dorsal attention systems [13, 14], including the anterior cingulate cortex and the dorsolateral prefrontal cortex (DLPFC) [15]. Individuals with high cognitive reserve seem to compensate for brain pathology by recruiting the contralateral DLPFC and by shifting brain networks from the posterior to anterior region $[5,8,16]$. Moreover, individuals with high cognitive reserve can tolerate more atrophy in the primary network before engaging a secondary, compensatory network [4]. Better functional connectivity between brain areas [8, 17] and increased frequency of high resting-state global functional connectivity [18] also seem to play a role, although the exact mechanisms are unclear at this point. Nevertheless, several theories have been proposed to account for these mechanisms. The scaffolding theory of aging and cognition claims that cognitive reserve represents the capacity to adapt to structural alterations by functional reorganization $[3,19]$. The compensation-related utilization of neural circuits hypothesis (CRUNCH) suggests that cognitive reserve is the capacity to use neural circuits efficiently to compensate for those that are impaired [19], whereas the model on hemispheric asymmetry reduction in older adults (HAROLD) suggests that cognitive reserve may only be the capacity to use additional brain circuits [19]. To characterize the compensational process and the underlying mechanism, we first need to understand the cognitive processes of cognitive reserve.

\subsection{Attributes of Cognitive Processes of Cognitive Reserve}

Cognitive reserve is an active process of counteracting the effects of brain pathology based on either compensatory strategies [6, 20] or alternate cognitive strategies $[1,21]$. It is an entirely functional process [12]. The critical threshold of brain pathology at which symptomatology can be withheld differs between individuals [1]. Thus, cognitive reserve capacities differ between individuals and are depleted over time with progressive pathology [2, 22]. Importantly, cognitive reserve cannot be explained by genetic factors [23] but is acquired through a lifetime of 
intellectual activities $[3,21]$, such as education, cognitive training, and participation in intellectual pursuits [24]. Thus, it is modifiable [12] and can be increased through interventions [25]. Education seems to be a factor contributing to a better cognitive reserve [7, 26, 27]. Studies have shown that high occupational demands preserve cognitive functioning and reduce dementia risk, indicating that occupational activities designed to challenge executive cognitive functions and information processing abilities also seem to increase cognitive reserve [28-30]. Cognitive interventions designed to improve reasoning abilities (in contrast to processing speed, memory, and no intervention) may also contribute to a better cognitive reserve because they not only improve cognitive functioning, but also significantly improve functioning in daily life (i.e., diminish dementia symptomatology [31, 32]). Recent studies suggest that bilingualism also contributes to cognitive reserve [33]. Accordingly, it is likely that intellectual activities that stimulate the use of higher cognitive abilities, such as executive functioning and reasoning, contribute to better maintenance of brain function. It is also likely that these cognitive abilities are critically involved in the mechanisms necessary for coping with brain damage.

\subsection{Findings on Cognition in Population-based Studies}

Since, by definition, cognitive reserve is 'software' that operates on a neural basis, it is cognitive processes operating in the brain that allow better cognitive function and fewer clinical symptoms in some individuals. Investigations of the role of factors such as education on cognitive reserve indicated that the cognitive processes of cognitive reserve are related to verbal memory and executive cognitive functioning. Longitudinal community-based studies of individuals aged 65+ showed that higher education was associated with superior performance in tests of verbal knowledge [34] as well as better performance in information processing $[35,36]$. Similar results were obtained in a study conducted in a cohort of individuals aged between 20 and 65 years [37]. Results from the British 1946 Birth Cohort confirmed associations between a higher level of education and better performance in verbal memory and processing abilities [38]. A meta-analysis of the effects of cognitive leisure activities on cognitive reserve also suggested that superior performance in verbal memory, executive functioning, and processing speed may play a role in the cognitive processes of cognitive reserve [39]. Evaluation of mental work demands also suggested that better verbal memory contributes to cognitive reserve [40, 41]. Findings from two population-based longitudinal cohort studies conducted in Japan and the UK emphasized that complex work with people is particularly associated with a higher performance in verbal memory tasks $[42,43]$. There is insufficient published evidence to conclude that mental work demands affect executive abilities. Bilingualism, another factor contributing to cognitive reserve, is wellknown to enhance executive abilities [44]. However, each of these studies investigated only the cognitive outcome. When taking into account brain pathology, such as white matter hyperintensities, education seems to be the main factor associated with better executive functioning in old age $[45,46]$.

\subsection{Cognitive Findings in Patient Studies}

The most important characteristic of cognitive reserve is that it preserves cognitive performance despite brain pathology. Studies in patients with $A D$, and thus with brain pathology, show a faster decline in individuals with a higher level of education (e.g., findings from a 
community-based multi-ethnic prospective cohort study in New York [47] and findings from patients at a French memory center [48]), suggesting that once cognitive reserve is depleted, the pathology is already so advanced that cognitive function declines rapidly. Only a few studies have investigated the decline of specific cognitive domains. In a Japanese hospital, AD patients who had a high level of education maintained superior verbal fluency (category) despite clinical symptoms [49]. Findings of a longitudinal population study in Southern France (PAQUID) revealed that, despite clinical dementia symptomatology, a higher level of education was associated with better performance in the Stroop effect and verbal learning tests [50]. Based on those findings, it can be speculated that better verbal memory and executive functioning might be key resources of cognitive reserve. Regarding bilingualism, one patient study on cognitive decline in bilingual dementia patients revealed that the nondominant language is a resource for the cognitive reserve when the dominant language is compromised [51]. Hence, it seems that AD patients with dementia actively compensate for lost memories by searching for semantic alternatives. Overall, however, there is only a limited number of studies investigating specific cognitive abilities (as opposed to global cognitive functioning) in patients. A major criterion of cognitive reserve is that individuals do not show clinical symptoms despite pathology; therefore, studies in which participants are all diagnosed with AD will not be particularly informative.

\section{Previous Theories on the Cognitive Processes of Cognitive Reserve}

To date, only one model related to cognitive processes in cognitive reserve has been published. 'Residual memory variance' represents the discrepancy between an individual's predicted and actual memory performance [22]. This residual correlates with verbal intelligence [52] and resilience to dementia [2], but not with indicators of brain pathology [52]. It is also associated with the rate of decline in executive function [53], highlighting the idea that memory and executive function play a key role in cognitive reserve. Although 'residual memory variance' is a useful approximation of cognitive reserve, this is limited by the dependence of the residual in memory performance on a demography-related average (i.e., the residual is derived by comparing the performance between individuals in the sample) and it has it been questioned whether cognitive reserve is just a deviation from the norm. Furthermore, this approximation infers that cognitive reserve is merely memory, while evidence implies that this is not the case [54]. Hence, the 'residual memory variance' model serves as a good estimate of cognitive reserve but does not explain the underlying mechanisms.

Other researchers suggest that the cognitive processes of cognitive reserve involve strategies for the use of memories and knowledge. Zahodne et al. (2015) proposed that conscious strategies may help older adults compensate for brain damage [2]. Lövdén et al. (2012) suggested that people use knowledge-based strategies to perform daily tasks efficiently [55]. Aronov et al. (2015) suggested that better cognitive functioning may be related to an increased use of external memory strategies [56]. The use of strategies is a concept that is common to all those theories. Executive functioning is the major cognitive ability required to develop and employ these strategies. As outlined in the previous section, executive functioning and verbal memory are superior in individuals with a higher level of education. Accordingly, it is reasonable to assume that executive functions play a role in cognitive reserve. However, most studies involved tests of verbal memory, while the relevance of implicit or procedural memory was not investigated. Even 
assuming that better verbal memory and executive functioning enhance the ability of individuals to cope with brain damage, the mechanism underlying this advantage remains to be elucidated.

\subsection{Memories and Mental Representations in Cognitive Reserve}

Verbal memories, or memories of any kind, guide behavior in everyday life. They allow us to recognize things and to make sense of the world. Therefore, it is not surprising that better verbal memory predicts a greater ability to manage the activities of daily life [57]. Memories are mental representations of the world and contain information on attributes, actions, shape, and categories $[58,59]$. Mental representations are connected in networks of information constructed by experience (see example Figure 1). They can be retrieved or activated, and decay if not used [60]. Combinations of mental representations in webs or networks are also referred to as a web of connections, semantic webs, schema, mental lexicons, and mental models, etc. Each of these terms describes how mental representations reflect structural relations between parts of the real world and are based on a theoretical construct that predicts behavior [59, 61]. An important aspect of these networks is that one mental representation acts as a cue that activates associated mental representations through priming. Priming occurs when perceiving or thinking about one thing activates semantically-related mental representations [62]. If memories decay due to dementia pathology, individuals can still use other mental representations in the network to which the original mental representation was once connected as a reference. Meta-analysis of experimental studies have shown that prior knowledge (as represented in networks of mental representations) serves as a schematic support that aids memory recall in older adults [63], in some cases enabling a memory performance similar to that of younger adults [64]. Individuals with more exhaustive connections between mental representations may thus, be able to compensate for lost representations. Networks of mental representations are experiencedependent; therefore, the level and content of education can be used to build up this type of knowledge base [65-67]. Other intellectual activities can have the same effect. For instance, physicians build up mental representations of medical diagnostic knowledge [68], engineers build up mental representations of mechanical devices [69], and musicians build up mental representations of musical meters [70].

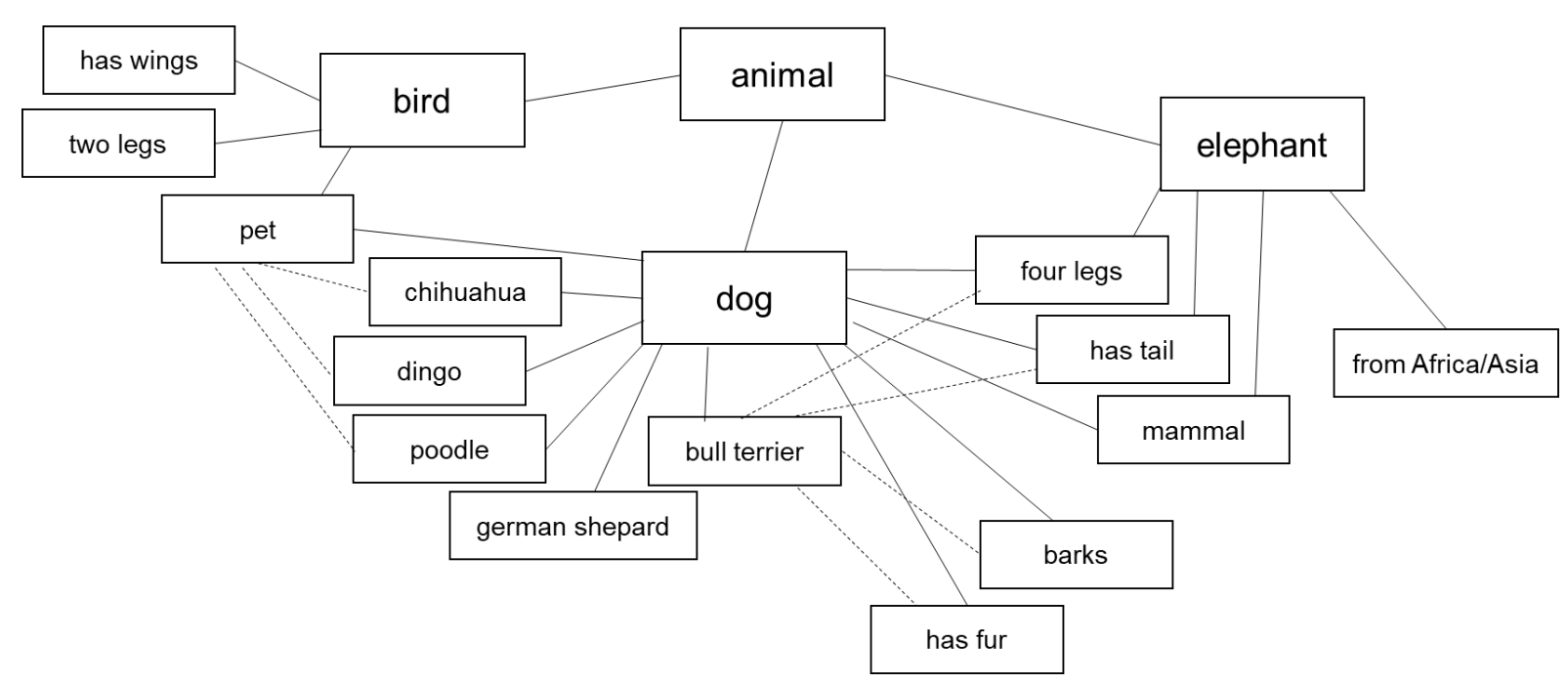

Figure 1 Network of mental representations 
The importance of networks of knowledge for dealing with memory deficiencies has already been demonstrated in studies with amnestic patients. Although amnestic patients cannot recall certain information, they can recognize information, respond to retrieval cues, and show effects of proactive interference [71, 72]. The use of representations that are associated in the network could explain the relatively high level of brain activity in the elderly. This enhanced brain activity, a form of 'over-activation', may appear to be nonselective and redundant [73], yet it helps people to rebuild the lost mental representation. As indicated in a review by Bullmore and Sporns (2009), studies of brain networks have shown that, in elderly individuals with structural brain perturbations, over-connectedness is necessary to provide the same level of efficiency as in the brains of younger individuals [74]. Over-activation has been observed as a sign of pathological dedifferentiation that occurs in the aging brain. Even so, extended networks of mental representations can help people deal with their daily activities. In fact, mental representations seem to be resistant to the effects of age. Older adults tend to have a greater knowledge of the meanings of words and maintain these expert skills as well as their semantic priming effects for the duration of a very long life [75]. Accordingly, mental representations are good candidates as contributors to cognitive reserve.

Networks of mental representations have additional advantages in the face of a degenerating brain. Mental models help individuals to process information more efficiently because they reduce the working memory load [76]. When working memory declines - due to age or pathology mental models may compensate for that. Organized mental representations are also fundamental to human reasoning. They contain information on conditionals, categories, probabilities, and causal relations [77]. People extend mental models to the real world by using principles of equiprobability and proportionality [78]. This helps them to make accurate judgments on real world problems and adapt behavior efficiently. Hence, if a mental representation decays (as in dementia), associated mental representations that reflect rules on conditionals, categories, or probabilities may help individuals to guess what the right behavior should be. Education can help to build up networks of mental representations that are useful to successfully manage life. Useful networks of mental representations could, for instance, be manifested in superior reasoning skills [79]. Education is thought to be a major contributor to cognitive reserve; therefore, mental representations may constitute the underlying mechanism.

\subsection{Executive Functioning in Cognitive Reserve}

By default, people with a better verbal memory have more elaborate networks of mental representations, which are also necessary to perform daily tasks. However, to deal with lost memories, it is necessary to reconnect, identify, or compensate for deficits. This is achieved by executive functioning. Executive functions are "control processes responsible for planning, assembling, coordinating, sequencing, and monitoring other cognitive operations [80]", such as recalling memories, problem-solving [81], decision-making, and logical reasoning [82]. Erroneous reasoning, for instance, is instigated by limitations in executive resources [83]. Empirically, better executive functioning is associated with less impairments in the activities of daily life [84] even after taking into account the effects of age and education [85]. In fact, executive functioning abilities moderate differences in memories related to accomplishing the activities of daily life [86]. Individuals with a higher level of education have a less rapid decline in executive functioning 
during the onset of $A D$ [87], suggesting that they are able to maintain better executive functioning despite the progression of pathology. AD patients with higher levels of education were similarly able to maintain a higher level of executive cognitive functioning than $A D$ patients with poor education [50]. The important role of education in the mechanism responsible for cognitive reserve is supported by the observation that, in more highly educated individuals, executive functions seem to be relatively resilient to $A D$ disease progression. Moreover, executive functioning is a core cognitive domain affected in $A D$; emphasizing the connection between $A D$ symptomatology and the level of executive abilities. Thus, individuals with better executive abilities are less likely to be diagnosed with $A D$, another fact that supports the importance of executive functions in mechanism of cognitive reserve.

The precise location of executive functioning in the brain is difficult to establish. The frontoparietal network appears to be a key site of executive functions [88], but different aspects of executive functioning, such as inhibition, maintenance, or monitoring, can be assigned to completely different regions of the brain [89]. Reasoning, for instance, is a concerted co-operation between several distinct brain areas [90] involving the anterior prefrontal, prefrontal, and right posterior parietal cortices [91] as well as the anterior insula, ventrolateral and DLPFC [92, 93]. Logical reasoning has the particular characteristic that it requires shifting brain networks [94]. The capacity to shift brain networks could be beneficial in the face of brain damage. Looking directly at strategies for encoding memories, brain imaging studies indicate that the brain areas used vary significantly between individuals $[88,95]$. The difficulty in assigning executive functioning a specific brain area indicates that this ability may be more resilient to brain damage and play a significant role in cognitive reserve.

\section{Cognitive Processes of Cognitive Reserve}

Based on available research findings, cognitive processes of cognitive reserve may involve mental representations and their associated networks, as well as executive functions (Table 1). Mental representations are the memories and knowledge that individuals have acquired during their lifetime and that they need in order to cope with daily life. Mental representation networks consist of connections between numerous mental representations and their features, which individuals can use either to rebuild a lost mental representation or, at least, to guess the appropriate behavior. Executive functioning represents the strategy by which individuals cope cognitively with lost mental representations. Executive functions are necessary to make sense of the 'incomplete' activation pattern and to produce an adequate behavior, for instance by using analogous or logical reasoning or developing a compensation strategy. An example is illustrated graphically in Figure 2. The concept emphasizes that cognitive reserve is the combination of both an elaborate network of mental representations and effective executive functioning. An elaborate network of mental representations alone is not useful because executive functioning is required to make sense of the remaining information. Similarly, executive functioning alone is not useful because there is insufficient information to rebuild an idea of the lost mental representation - like a cook with an empty fridge.

An important aspect of this conceptual approach to cognitive reserve is that the 'magnitude' of cognitive reserve depends on the characteristics of mental representations. Environmental factors such as education, and occupational and leisure activities build up mental representations, which 
are reinforced by re-activation of mental representations in later life (e.g., in leisure activities or cognitive training). This concept predicts that individuals with more elaborate networks of mental representations have a higher cognitive reserve and that cognitive reserve is depleted by damage to these mental representations. Moreover, the concept also predicts that cognitive reserve depends on cognitive strategies acquired throughout the lifetime of an individual. Interventions that enhance executive functions should improve the capacity to tolerate brain damage compared to those that improve only working memory or processing speed. Similarly, the concept predicts that cognitive reserve is reduced by disorders associated with impaired executive functioning, such as depression [96] or schizophrenia [97]. Other factors that influence cognition in old age, such as genetics, vascular health, physical activity, and diet also influence cognitive health [98] and brain systems including structure, function and neurochemistry [99] and could therefore, also influence the cognitive abilities associated with cognitive reserve.

Table 1 Quick facts

\begin{tabular}{ll}
\hline Concept & Attributes of the concept \\
\hline Cognitive reserve & Capacity for normal cognitive function despite brain damage \\
\hline & Active mechanism for coping with brain damage \\
& Threshold differs between individuals \\
\hline & Depleted with progressing pathology \\
\hline Acquired through intellectual activities and can be modified \\
\hline Memories that reflect parts of the real world \\
\hline Comprise information on attributes, actions, shape, categories, etc. \\
\hline $\begin{array}{l}\text { Constructed by experience, re-activated by use, and decay if not } \\
\text { used }\end{array}$ \\
\hline $\begin{array}{l}\text { Resistant to aging } \\
\text { Connected to other mental representations in a network, such as } \\
\text { schemas, mental models, semantic webs and others }\end{array}$ \\
\hline $\begin{array}{l}\text { Cued by activation of connected mental representations in the } \\
\text { network (priming) }\end{array}$ \\
\hline \begin{tabular}{l} 
Mental control processes, such as planning, decision-making, and \\
reasoning \\
of brain networks \\
\hline
\end{tabular} \\
\hline Associated with less impairment in daily life despite brain pathology \\
\hline
\end{tabular}


VISUAL STIMULI

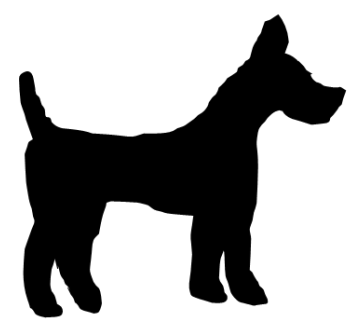

ACTIVATED MENTAL REPRESENTATIONS CONNECTED TO LOST MEMORY

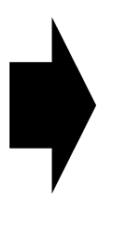

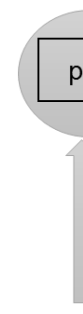

Executive functions select most relevant concept to initiate behavioral response

\section{BEHAVIORAL} RESPONSE

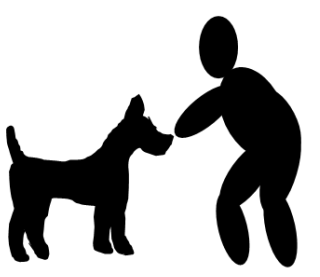

Figure 2 Processing of stimuli through a network of mental representations and executive functioning in the face of lost memories - possible mechanism of cognitive reserve. Black box $=$ lost memory

More refined measures of brain structure and function are needed to explain how executive cognitive abilities compensate for brain pathology. Models of neurocognitive aging (CRUNCH, HAROLD, scaffolding, dedifferentiation, see also Section 3 and [100]) must be extended to address characteristics of functional connectivity. Connectivity has been shown to be an important factor in maintaining cognitive performance in old age [101] and may be more relevant to understanding human cognition than blood-oxygen level-dependent (BOLD) signals alone [102]. BOLD signals in fMRI imaging do not necessarily reflect differences in neuronal processing and can be misinterpreted [103], especially when studying age-related differences [103, 104]. Techniques that offer a higher temporal resolution of electrophysiological signals could reveal interactions between cortical brain areas [105] that might be core processes involved in cognitive reserve. It is likely that the complex interplay of executive cognitive abilities with mental representations in the face of brain pathology cannot be revealed by current imaging techniques and more temporal and locally sensitive techniques are required.

\section{Conclusion}

Although the possible neurobiological characteristics of cognitive reserve have been investigated by several researchers $[3,25]$, the cognitive processes that help some individuals maintain a better level of functioning despite brain damage remain to be identified. Here, we have outlined the findings on cognitive processes that seem to be involved in cognitive reserve, and indicate that cognitive reserve may be the result of a combination of elaborate networks of mental representations and proficient executive functioning. On the one hand, this conceptual approach provides attributes that can be tested and, on the other hand, permits the prediction of an outcome given certain attributes. Accordingly, our conceptual approach provides the necessary characteristics for a conceptual model of cognitive reserve [13].

Further studies are required to test the assumptions of the concept and refine the components of the proposed concept. Table 2 outlines some of the important outstanding questions and future directives. Therein, false non-significant effects are just as important as significant findings 
because they clarify the cognitive processes that help some individuals to maintain a better level of cognitive functioning. Evidently, models of neurocognitive aging need to be extended to incorporate specific cognitive abilities that are measurable and describe how they relate to functional connectivity. To investigate cognitive processes at a neurological level with respect to the progression of $A D$ pathology, new techniques with a higher temporal resolution are required. Carefully controlled experimental designs that combine rest and task data from different modalities [106] can then reveal information on the interplay of executive cognitive abilities with mental representations in the face of brain pathology.

Table 2 Outstanding questions and future directives

\section{Outstanding questions and future directives}

Does the 'magnitude' of cognitive reserve correspond to the density of mental representations, and is it depleted when most of the mental representations are lost?

What are the features of mental models, schemas, semantic webs, etc. in individuals with high compared to low cognitive reserve?

How does cueing or priming of associated mental representations affect behavioral response in cases of brain damage?

How does education and other lifetime experiences build 'useful' networks of mental representations and what characteristics make them useful in the damaged brain?

Does the re-activation of mental representations in later life - e.g., through leisure activities or cognitive training - increase cognitive reserve in a dose-dependent manner?

Are elaborated networks of mental representations a means to boosting working memory (and can this compensate for loss in processing speed in old age)?

What exactly do we mean by executive functioning (inhibition, monitoring, selection, sustained attention, reasoning, etc.) and how does this impact cognitive reserve?

Do interventions designed to improve executive functions enhance cognitive reserve more than those designed to improve working memory or processing speed? Do interventions designed to improve other cognitive strategies, such as regulation of emotion and social skills contribute to a specific cognitive reserve in that area of daily life?

Is the capacity to shift brain networks a key characteristic of proficient executive functioning and does this capacity predict a better cognitive performance in the face of brain damage?

Do disorders that damage executive functioning also damage cognitive reserve?

\section{Author Contributions}

FSR conducted the literature review, conceive and wrote the paper.

\section{Funding}

FSR reports a grant from the German Research Foundation (DFG): grant \# TH2137/3-1.

\section{Competing Interests}

The author reports no conflict of interest. 


\section{References}

1. Stern Y. What is cognitive reserve? Theory and research application of the reserve concept. J Int Neuropsychol Soc. 2002; 8: 448-460.

2. Zahodne LB, Manly JJ, Brickman AM, Narkhede A, Griffith EY, Guzman VA, et al. Is residual memory variance a valid method for quantifying cognitive reserve? A longitudinal application. Neuropsychologia. 2015; 77: 260-266.

3. Barulli D, Stern Y. Efficiency, capacity, compensation, maintenance, plasticity: Emerging concepts in cognitive reserve. Trends Cogn Sci. 2013; 17: 502-509.

4. Steffener J, Stern Y. Exploring the neural basis of cognitive reserve in aging. Biochimica et Biophysica Acta (BBA)-Molecular Basis of Disease. 2012; 1822: 467-473.

5. Bartres-Faz D, Arenaza-Urquijo EM. Structural and functional imaging correlates of cognitive and brain reserve hypotheses in healthy and pathological aging. Brain Topogr. 2011; 24: 340357.

6. Ferrari C, Nacmias B, Bagnoli S, Piaceri I, Lombardi G, Pradella S, et al. Imaging and cognitive reserve studies predict dementia in presymptomatic Alzheimer's disease subjects. NeuroDegenerative Diseases. 2014; 13: 157-159.

7. Modica CM, Bergsland N, Dwyer MG, Ramasamy DP, Carl E, Zivadinov R, et al. Cognitive reserve moderates the impact of subcortical gray matter atrophy on neuropsychological status in multiple sclerosis. Mult Scler. 2016; 22: 36-42.

8. Morbelli S, Nobili F. Cognitive reserve and clinical expression of Alzheimer's disease: Evidence and implications for brain PET imaging. American Journal of Nuclear Medicine and Molecular Imaging. 2014; 4: 239.

9. Almeida RP, Schultz SA, Austin BP, Boots EA, Dowling NM, Gleason CE, et al. Effect of Cognitive Reserve on Age-Related Changes in Cerebrospinal Fluid Biomarkers of Alzheimer Disease. JAMA Neurol. 2015; 72: 699-706.

10. Bennett DA, Schneider JA, Wilson RS, Bienias JL, Arnold SE. Education modifies the association of amyloid but not tangles with cognitive function. Neurology. 2005; 65: 953-955.

11. Rolstad S, Nordlund A, Eckerstrom C, Gustavsson MH, Zetterberg H, Wallin A. Cognitive reserve in relation to abeta42 in patients converting from $\mathrm{MCl}$ to dementia - a follow-up report. Dement Geriatr Cogn Disord. 2009; 28: 110-115.

12. Liao YC, Liu RS, Teng EL, Lee YC, Wang PN, Lin KN, et al. Cognitive reserve: A SPECT study of 132 Alzheimer's disease patients with an education range of 0-19 years. Dement Geriatr Cogn Disord. 2005; 20: 8-14.

13. Medaglia JD, Pasqualetti F, Hamilton RH, Thompson-Schill SL, Bassett DS. Brain and cognitive reserve: Translation via network control theory. Neurosci Biobehav Rev. 2017; 75: 53-64.

14. Bozzali M, Dowling C, Serra L, Spano B, Torso M, Marra C, et al. The impact of cognitive reserve on brain functional connectivity in Alzheimer's disease. J Alzheimers Dis. 2015; 44: 243-250.

15. Colangeli S, Boccia M, Verde P, Guariglia P, Bianchini F, Piccardi L. Cognitive reserve in healthy aging and Alzheimer's disease: A Meta-Analysis of fMRI studies. Am J Alzheimers Dis Other Demen. 2016; 31: 443-449. 
16. Bastin C, Yakushev I, Bahri MA, Fellgiebel A, Eustache F, Landeau B, et al. Cognitive reserve impacts on inter-individual variability in resting-state cerebral metabolism in normal aging. Neuroimage. 2012; 63: 713-722.

17. Franzmeier N, Buerger K, Teipel S, Stern Y, Dichgans M, Ewers M, Alzheimer's disease neuroimaging $\mathrm{I}$. Cognitive reserve moderates the association between functional network anti-correlations and memory in $\mathrm{MCl}$. Neurobiol Aging. 2017; 50: 152-162.

18. Franzmeier N, Caballero MA, Taylor AN, Simon-Vermot L, Buerger K, Ertl-Wagner B, et al. Resting-state global functional connectivity as a biomarker of cognitive reserve in mild cognitive impairment. Brain Imaging Behav. 2016. doi:10.1007/s11682-016-9599-1.

19. Borroni B, Premi E, Bozzali M, Padovani A. Reserve mechanisms in neurodegenerative diseases: From bench to bedside and back again. Curr Med Chem. 2012; 19: 6112-6118.

20. Nucci $M$, Mapelli D, Mondini S. Cognitive Reserve Index questionnaire (CRIq): A new instrument for measuring cognitive reserve. Aging Clin Exp Res. 2012; 24: 218-226.

21. Schwartz CE, Rapkin BD, Healy BC. Reserve and Reserve-building activities research: Key challenges and future directions. BMC Neurosci. 2016; 17: 62.

22. Zahodne LB, Manly JJ, Brickman AM, Siedlecki KL, DeCarli C, Stern Y. Quantifying cognitive reserve in older adults by decomposing episodic memory variance: Replication and extension. J Int Neuropsych Soc. 2013; 19: 854-862.

23. Robertson $\mathrm{IH}$. A noradrenergic theory of cognitive reserve: Implications for Alzheimer's disease. Neurobiol Aging. 2013; 34: 298-308.

24. Grotz C, Seron X, van Wissen M, Adam S. How should proxies of cognitive reserve be evaluated in a population of healthy older adults? Int Psychogeriatr. 2017; 29: 123-136.

26. Bonner-Jackson A, Long JD, Westervelt H, Tremont G, Aylward E, Paulsen JS, et al. Cognitive reserve and brain reserve in prodromal Huntington's disease. J Int Neuropsych Soc. 2013; 19: 739-750.

27. Summers MJ, Thow ME, Ward DD, Saunders NL, Klekociuk SZ, Imlach AR, et al. Validation of a dynamic measure of current cognitive reserve in a longitudinally assessed sample of healthy older adults. Assessment. 2017. doi:10.1177/1073191116685806.

28. Then FS, Luppa M, Schroeter ML, Konig HH, Angermeyer MC, Riedel-Heller SG. Enriched environment at work and the incidence of dementia: Results of the leipzig longitudinal study of the aged (LEILA 75+). Plos One. 2013; 8.

29. Then FS, Luck T, Luppa M, Konig HH, Angermeyer MC, Riedel-Heller SG. Differential effects of enriched environment at work on cognitive decline in old age. Neurology. 2015; 84: 21692176.

30. Then FS, Luck T, Heser K, Ernst A, Posselt T, Wiese B, et al. Which types of mental work demands may be associated with reduced risk of dementia? Alzheimers Dement. 2016. doi:10.1016/j.jalz.2016.08.008.

31. Willis SL, Tennstedt SL, Marsiske M, Ball K, Elias J, Koepke KM, et al. Long-term effects of cognitive training on everyday functional outcomes in older adults. Jama. 2006; 296: 28052814.

32. Rebok GW, Ball K, Guey LT, Jones RN, Kim HY, King JW, et al. Ten-year effects of the advanced cognitive training for independent and vital elderly cognitive training trial on cognition and everyday functioning in older adults. J Am Geriatr Soc. 2014; 62: 16-24. 
33. Guzmán-Vélez E, Tranel D. Does bilingualism contribute to cognitive reserve? cognitive and neural perspectives. Neuropsychology. 2015; 29: 139-150.

34. Christensen $\mathrm{H}$, Korten $\mathrm{AE}$, Jorm AF, Henderson AS, Jacomb PA, Rodgers $\mathrm{B}$, et al. Education and decline in cognitive performance: Compensatory but not protective. Int J Geriatr Psych. 1997; 12: 323-330.

35. Le Carret N, Lafont S, Mayo W, Fabrigoule C. The effect of education on cognitive performances and its implication for the constitution of the cognitive reserve. Dev Neuropsychol. 2003; 23: 317-337.

36. Salthouse TA. What do adult age differences in the Digit Symbol Substitution Test reflect? J Gerontol. 1992; 47: P121-P128.

37. Roldan-Tapia L, Garcia J, Canovas R, Leon I. Cognitive reserve, age, and their relation to attentional and executive functions. Appl Neuropsychol. 2012; 19: 2-8.

38. Hatch SL, Feinstein L, Link BG, Wadsworth ME, Richards M. The continuing benefits of education: Adult education and midlife cognitive ability in the British 1946 birth cohort. J Gerontol. 2007; 62: S404-S414.

39. Yates LA, Ziser S, Spector A, Orrell M. Cognitive leisure activities and future risk of cognitive impairment and dementia: Systematic review and meta-analysis. Int Psychogeriatr. 2016; 28: 1791-1806.

40. Finkel D, Andel R, Gatz M, Pedersen NL. The role of occupational complexity in trajectories of cognitive aging before and after retirement. Psychol Aging. 2009; 24: 563-573.

41. Fisher GG, Stachowski A, Infurna FJ, Faul JD, Grosch J, Tetrick LE. Mental work demands, retirement, and longitudinal trajectories of cognitive functioning. J Occup Health Psychol. 2014; 19: 231-242.

42. Ishioka $Y$, Gondo $Y$, Masui $Y$, Nakagawa $T$, Tabuchi $M$, Ogawa $M$, et al. Occupational complexity and late-life memory and reasoning abilities. Shinrigaku Kenkyu. 2015; 86: 219229.

43. Smart EL, Gow AJ, Deary IJ. Occupational complexity and lifetime cognitive abilities. Neurology. 2014; 83: 2285-2291.

44. Grant A, Dennis NA, Li P. Cognitive control, cognitive reserve, and memory in the aging bilingual brain. Frontiers in Psychology. 2014; 5: 1401.

45. Kaplan RF, Cohen RA, Moscufo N, Guttmann C, Chasman J, Buttaro M, et al. Demographic and biological influences on cognitive reserve. J Clin Exp Neuropsychol. 2009; 31: 868-876.

46. Siedlecki KL, Stern Y, Reuben A, Sacco RL, Elkind MS, Wright CB. Construct validity of cognitive reserve in a multiethnic cohort: The Northern Manhattan Study. J Int Neuropsychol Soc. 2009; 15: 558-569.

47. Scarmeas N, Albert SM, Manly JJ, Stern Y. Education and rates of cognitive decline in incident Alzheimer's disease. J Neurol Neurosurg Psychiatry. 2006; 77: 308-316.

48. Bruandet A, Richard F, Bombois S, Maurage CA, Masse I, Amouyel P, et al. Cognitive decline and survival in Alzheimer's disease according to education level. Dement Geriatr Cogn Disord. 2008; 25: 74-80.

49. Kawano N, Umegaki H, Suzuki Y, Yamamoto S, Mogi N, Iguchi A. Effects of educational background on verbal fluency task performance in older adults with Alzheimer's disease and mild cognitive impairment. Int Psychogeriatr. 2010; 22: 995-1002. 
50. Le Carret N, Auriacombe S, Letenneur L, Bergua V, Dartigues JF, Fabrigoule C. Influence of education on the pattern of cognitive deterioration in $A D$ patients: The cognitive reserve hypothesis. Brain Cognition. 2005; 57: 120-126.

51. Kowoll ME, Degen C, Gladis S, Schröder J. Neuropsychological profiles and verbal abilities in lifelong bilinguals with mild cognitive impairment and Alzheimer's disease. J Alzheimers Dis. 2015; 45: 1257-1268.

52. Habeck C, Razlighi Q, Gazes Y, Barulli D, Steffener J, Stern Y. Cognitive Reserve and Brain Maintenance: Orthogonal Concepts in Theory and Practice. Cerebral cortex. 2016. doi:10.1093/cercor/bhw208.

53. Reed BR, Mungas D, Farias ST, Harvey D, Beckett L, Widaman K, et al. Measuring cognitive reserve based on the decomposition of episodic memory variance. Brain. 2010; 133: 21962209.

54. Serra L, Bruschini M, Di Domenico C, Gabrielli GB, Marra C, Caltagirone C, et al. Memory is not enough: The neurobiological substrates of dynamic cognitive reserve. J Alzheimers Dis. 2017. doi:10.3233/JAD-170086.

55. Lovden M, Brehmer $\mathrm{Y}$, Li SC, Lindenberger U. Training-induced compensation versus magnification of individual differences in memory performance. Front Hum Neurosci. 2012; 6: 141. doi:10.3389/fnhum.2012.00141.

56. Aronov A, Rabin LA, Fogel J, Chi SY, Kann SJ, Abdelhak N, et al. Relationship of cognitive strategy use to prospective memory performance in a diverse sample of nondemented older adults with varying degrees of cognitive complaints and impairment. Neuropsychol Dev Cogn B Aging Neuropsychol Cogn. 2015; 22: 486-501.

57. Mograbi DC, Faria Cde A, Fichman HC, Paradela EM, Lourenco RA. Relationship between activities of daily living and cognitive ability in a sample of older adults with heterogeneous educational level. Ann Indian Acad Neurol. 2014; 17: 71-76.

58. Sternberg RJ. Introduction to optimizing learning in college: Tips from cognitive psychology. Perspect Psychol Sci. 2016; 11: 651. doi:10.1177/1745691616672227.

59. Sima JF, Schultheis H, Barkowsky T. Differences between spatial and visual mental representations. Front Psychol. 2013. doi:10.3389/fpsyg.2013.00240.

60. Smith R, Queller S. Mental Representations. In: Tesser A, Schwarz N, editors. Blackwell Handbook of Social Psychology: Intraindividual Processes. Malden, Massachusetts: Blackwell Publisher Ltd; 2001.

61. Copeland DE, Radvansky GA. Aging and integrating spatial mental models. Psychol Aging. 2007; 22: 569-279.

62. Grady CL, Craik FI. Changes in memory processing with age. Curr Opin Neurobiol. 2000; 10: 224-231.

63. Castel AD. Memory for grocery prices in younger and older adults: The role of schematic support. Psychol Aging. 2005; 20: 718-721.

64. Old SR, Naveh-Benjamin M. Differential effects of age on item and associative measures of memory: a meta-analysis. Psychol Aging. 2008; 23: 104.

65. Mason RA, Just MA. Physics instruction induces changes in neural knowledge representation during successive stages of learning. Neuroimage. 2015; 111: 36-48.

66. Ashcraft $\mathrm{MH}$, Fierman BA. Mental addition in third, fourth, and sixth graders. J Exp Child Psychol. 1982; 33: 216-234. 
67. Van Oostendorp H, Goldman SR. The construction of mental representations during reading. Psychology Press; 1998.

68. Custers EJ, Regehr G, Norman GR. Mental representations of medical diagnostic knowledge: a review. Acad Med. 1996; 71: S55-S61.

69. Moss J, Kotovsky K, Cagan J. The role of functionality in the mental representations of engineering students: some differences in the early stages of expertise. Cogn Sci. 2006; 30: 65-93.

70. Palmer C, Krumhansl CL. Mental representations for musical meter. J Exp Psychol. 1990; 16: 728.

71. Hirst W, Johnson MK, Kim JK, Risse G, Phelps EA, Volpe BT. Recognition and Recall in Amnesiacs. Journal of experimental psychology. Learning, Memory, and Cognition. 1986; 12: 445-451.

72. Warrington EK, Weiskrantz L. A study of learning and retention in Amnesic patients. Neuropsychologia. 1968; 6: 283. doi:10.1016/0028-3932(68)90026-2.

73. Reuter-Lorenz P. New visions of the aging mind and brain. Trends Cogn Sci. 2002; 6: 394.

74. Bullmore E, Sporns O. Complex brain networks: Graph theoretical analysis of structural and functional systems. Nat Rev Neurosci. 2009; 10: 186-198. doi:10.1038/nrn2575.

75. Bialystok E, Craik FIM. Lifespan cognition: Mechanisms of Change: Oxford University Press; 2006.

76. Gilbert DK, Rogers WA. Age-related differences in the acquisition, utilization, and extension of a spatial mental model. J Gerontol. 1999; 54: P246-P255.

77. Ali N, Chater N, Oaksford $\mathrm{M}$. The mental representation of causal conditional reasoning: Mental models or causal models. Cognition. 2011; 119: 403-418.

78. Chevalley T, Schaeken W. Considering too few alternatives: The mental model theory of extensional reasoning. Q J Exp Psychol (Hove). 2016; 69: 728-751.

79. Johnson-Laird PN. Mental models and human reasoning. P Natl Acad Sci USA. 2010; 107: 18243-18250.

80. Salthouse TA, Atkinson TM, Berish DE. Executive functioning as a potential mediator of agerelated cognitive decline in normal adults. J Exp Psychol-General. 2003; 132: 566-594.

81. Brandt J, Aretouli E, Neijstrom E, Samek J, Manning K, Albert MS, et al. Selectivity of executive function deficits in mild cognitive impairment. Neuropsychology. 2009; 23: 607-618.

82. Goghari VM, Lawlor-Savage L. Comparison of cognitive change after working memory training and logic and planning training in healthy older adults. Front Aging Neurosci. 2017; 9: 39. doi:10.3389/fnagi.2017.00039.

83. Neys W de. Dual processing in reasoning: Two systems but one reasoner. Psychol Sci. 2006; 17: 428-433.

84. Vaughan L, Giovanello K. Executive function in daily life: Age-related influences of executive processes on instrumental activities of daily living. Psychol Aging. 2010; 25: 343-355.

85. McAlister C, Schmitter-Edgecombe M. Executive function subcomponents and their relations to everyday functioning in healthy older adults. J Clin Exp Neuropsychol. 2016; 38: 925-940.

86. Sauzeon H, N'Kaoua B, Pala PA, Taillade M, Auriacombe S, Guitton P. Everyday-like memory for objects in ageing and Alzheimer's disease assessed in a visually complex environment: The role of executive functioning and episodic memory. J Neuropsychol. 2016; 10: 33-58. 
87. Amieva $H$, Mokri $H$, Le Goff $M$, Meillon $C$, Jacqmin-Gadda $H$, Foubert-Samier $A$, et al. Compensatory mechanisms in higher-educated subjects with Alzheimer's disease: A study of 20 years of cognitive decline. Brain. 2014; 137: 1167-1175.

88. Barbey AK, Colom R, Solomon J, Krueger F, Forbes C, Grafman J. An integrative architecture for general intelligence and executive function revealed by lesion mapping. Brain. 2012; 135: 1154-1164.

89. Aron AR. Progress in executive-function research: From tasks to functions to regions to networks. Current Directions in Psychological Science. 2008; 17: 124-129.

90. Reverberi C, Bonatti LL, Frackowiak RS, Paulesu E, Cherubini P, Macaluso E. Large scale brain activations predict reasoning profiles. Neuroimage. 2012; 59: 1752-1764.

91. Fangmeier T, Knauff M, Ruff CC, Sloutsky V. FMRI evidence for a three-stage model of deductive reasoning. J Cogn Neurosci. 2006; 18: 320-334.

92. Yuan Z, Qin W, Wang D, Jiang T, Zhang Y, Yu C. The salience network contributes to an individual's fluid reasoning capacity. Behav Brain Res. 2012; 229: 384-390.

93. Khemlani SS, Barbey AK, Johnson-Laird PN. Causal reasoning with mental models. Front Hum Neurosci. 2014; 8: 849. doi:10.3389/fnhum.2014.00849.

94. Houde O, Zago L, Mellet E, Moutier S, Pineau A, Mazoyer B, et al. Shifting from the perceptual brain to the logical brain: The neural impact of cognitive inhibition training. J Cogn Neurosci. 2000; 12: 721-728.

95. Heiss WD, Pawlik G, Holthoff V, Kessler J, Szelies B. PET correlates of normal and impaired memory functions. Cerebrovasc Brain Metab Rev. 1992; 4: 1-27.

96. Snyder HR. Major depressive disorder is associated with broad impairments on neuropsychological measures of executive function: A meta-analysis and review. Psychological Bulletin. 2013; 139: 81-132.

97. Velligan DI, Bow-Thomas CC. Executive function in schizophrenia. Semin Clin Neuropsychiatry. 1999; 4: 24-33.

98. Plassman BL, Williams JW Jr, Burke JR, Holsinger T, Benjamin S. Systematic review: factors associated with risk for and possible prevention of cognitive decline in later life. Ann Intern Med. 2010; 153: 182-193.

99. Mattson MP, Chan SL, Duan W. Modification of brain aging and neurodegenerative disorders by genes, diet, and behavior. Physiol Rev. 2002; 82: 637- 672.

100. Grady C. The cognitive neuroscience of ageing. Nat Rev Neurosci. 2012; 13: 491-505.

101. Tsvetanov KA, Henson RN, Tyler LK, Razi A, Geerligs L, Ham TE, et al; Cambridge Centre for Ageing and Neuroscience. Extrinsic and intrinsic brain network connectivity maintains cognition across the lifespan despite accelerated decay of regional brain activation. J Neurosci. 2016; 36: 3115-3126.

102. Tsvetanov KA, Ye Z, Hughes L, Samu D, Treder MS, Wolpe N, et al; Cambridge Centre for Ageing and Neuroscience. Activity and connectivity differences underlying inhibitory control across the adult life span. J Neurosci. 2018; 38: 7887-7900.

103. Tsvetanov KA, Henson RN, Tyler LK, Davis SW, Shafto MA, Taylor JR, et al. The effect of ageing on $\mathrm{fMRI}$ : Correction for the confounding effects of vascular reactivity evaluated by joint $\mathrm{fMRI}$ and MEG in 335 adults. Hum Brain Mapp. 2015; 36: 2248-2269. 
104. Geerligs L, Tsvetanov KA, Cam-Can, Henson RN. Challenges in measuring individual differences in functional connectivity using fMRI: The case of healthy aging. Hum Brain Mapp. 2017; 38: 4125-4156.

105. Mandal PK, Banerjee A, Tripathi M, Sharma A. A comprehensive review of magnetoencephalography (MEG) studies for brain functionality in healthy aging and Alzheimer's disease (AD). Front Comput Neurosci. 2018; 12: 60.

106. Geerligs, L., Tsvetanov, K.A., 2017. The use of resting state data in an integrative approach to studying neurocognitive ageing-commentary on Campbell and Schacter (2016). Lang. Cogn. Neurosci. 32. doi:10.1080/23273798.2016.1251600

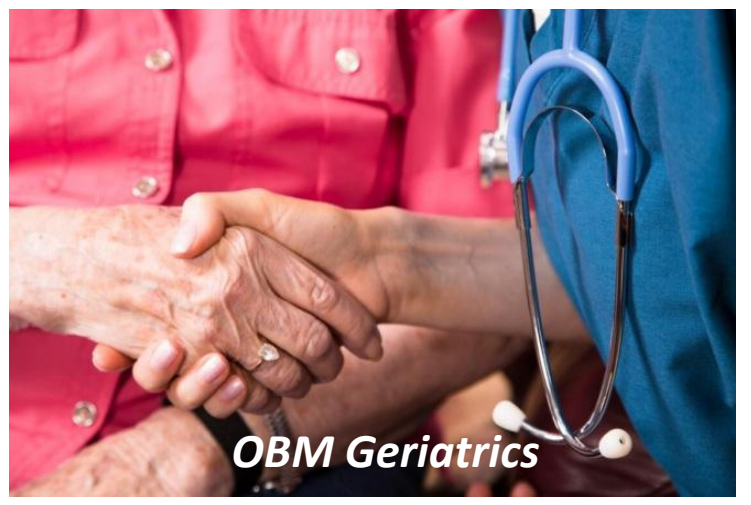

Enjoy $O B M$ Geriatrics by:

1. Submitting a manuscript

2. Joining in volunteer reviewer bank

3. Joining Editorial Board

4. Guest editing a special issue

For more details, please visit:

http://www.lidsen.com/journals/geriatrics 